\title{
AN OBSERVER-BASED FUSION METHOD USING MULTICORE OPTICAL SHAPE SENSORS AND ULTRASOUND IMAGES FOR MAGNETICALLY-ACTUATED CATHETERS
}

\author{
A. Denasi', F. Khan',2, K.J. Boskma', M. Kaya',2, C. Hennersperger ${ }^{3}$, R. Göbl3, M. Tirindelli3, N. \\ $\mathrm{Navab}^{3}$ and S. Misra ${ }^{1,2}$ \\ IUniversity of Groningen and University Medical Center Groningen, the Netherlands, 2University of Twente, \\ the Netherlands, 3Technical University of Munich, Germany. E-mail: a.denasi@umcg.nl
}

Kolff program: Restoring organ function by means of regenerative medicine (REGENERATE), Bioadhesion, biocompatibility and infection (BIOBI)

\section{Introduction}

Flexible medical instruments such as endoscopes, catheters and needles constitute an important set of tools used in minimally invasive surgery (MIS). The integration of robotic navigation with MIS is becoming increasingly popular due to the advances in sensing and actuation technologies. Remote actuation of flexible catheters by external magnetic fields is a favorable technique. Besides the actuation method, acquiring an accurate tip pose is crucial for the success of robotically-guided MIS procedures. The tip pose is often acquired using ultrasound (US) since, it has the advantage of being easily accessible, safe to operate and the possibility to image using a hand-held probe. Nonetheless, the visual tracking methods used to estimate the tip pose from the acquired US images are prone to failure due to the low signal-to-noise ratio and an excessive number of image artifacts. Besides image-based tracking, catheter poses can also be retrieved by common shape sensing techniques using fiber-optic sensors. In this study, the tip position obtained from transverse 2D ultrasound images and multicore optical shape sensors are combined using a robust sensor fusion algorithm.

\section{Methods}

The tip pose of the flexible catheter is obtained from the US images using two different algorithms, template-based tracking and convolutional neural networks. The template-based tracking algorithm registers the initial template image of the catheter and the current image. The training of the convolutional neural network is performed using stereo camera images as the ground truth. The 3D shape and consequently the tip pose of the catheter are also be obtained using sets of four Fiber Bragg Grating (FBG) sensors placed along the length of the catheter. The optical fiber used in this study contains four cores, where one of the cores is placed in the center axis of the fiber. The curvature and its direction at each sensor location is computed using the strain measurements. These are then interpolated to compute the tangent vector of the curve of the catheter. Consequently, the shape and respectively the tip pose of the flexible catheter is determined by numerical integration of the tangent vector. The tip position obtained both from the FBG sensors and US images are fused using two different algorithms, a Luenberger state observer and a Kalman filter. In both of these algorithms, it is assumed that the dynamics of the catheter can be approximated by a linear time-invariant model.

\section{Results}

Using the template-based tracker, the catheter tip is successfully tracked in long term using scaleoffset and affine motion models. Using the convolutional neural network an average error of $\mathrm{I} .4 \mathrm{II}$ $\mathrm{mm}$ is obtained when it is applied to an unseen image sequence. This implies, that for a more robust network, more data should be collected, covering different motion patterns and visual appearances of the catheter. The mean and standard deviation of the Euclidean error for the Luenberger observer is $0.2 \pm 0.11$ [mm] whereas for the Kalman filter it is $0.18 \pm 0.13$ [mm], respectively.

\section{Conclusions}

The template-based tracker performs slightly better compared to the convolutional neural network tracker in localizing the tip position of the catheter. The estimation performance of the Luenberger observer and Kalman filter are similar to each other. Future research considers investigating the online implementation of the algorithm with improvements for different trajectories. 